\section{Evolutionary Dynamics: evolutionary genetics with the genes taken out}

\author{
Evolutionary Dynamics: Exploring the Interplay \\ of Selection, Accident, Neutrality and Function \\ Edited by JP Crutchfield and P Schuster \\ Oxford University Press, New York; 2003. 452 pp. \\ $£ 29.95$, paperback. ISBN 0-19-5142659.
}

Heredity (2005) 94, 139-140. doi:10.1038/sj.hdy.6800535

\section{Reviewed by JFY Brookfield}

In October 1998 the Santa Fe Institute, as one of its series of conferences exploring multidisciplinary collaborations, held a meeting entitled 'Towards a Comprehensive Dynamics of Evolution - Exploring the Interplay of Selection, Accident, Neutrality and Function'. One result of this is a multi-author volume, characterised by sophisticated mathematical approaches to some of the unsolved problems of evolutionary theory. The main sections start with Macroevolution, where Niles Eldredge, and then Gunther Elbe, presents paleontological results showing patterns of morphological evolution. In the section 'Epochal Evolution', Aviv Bergman and Marc Feldman, followed by James Crutchfield and then Sergey Gavrilets, present theoretical results indicating why phenotypic evolution might be expected to show the patterns of constancy and sudden change seen in fossil records. There follow sections on Population Dynamics, Genetics, and Optimization, and on the Evolution of Cooperation. Notwithstanding the title of the third section, and the contribution here from Tomoko Ohta, there is far too little genetics in here for my taste genes are generally used as merely infinitely flexible explanatory variables for the phenotype. The actual molecular properties of genes, how they are expressed, and how they interact, are not generally seen as being required in the modelling process. Instead, the basis of the models presented seems to be just the principle of descent with mutational modification, with fitnesses being dependent, often in complex epistatic ways, on multilocus genotypes, but with these dependencies being arbitrary and not based on biological knowledge.

While there are many themes explored in this collection, one strong message emerging from the chapters in the first half is the synthesis of theories of evolutionary dynamics with paleontological observation of evolutionary trends. The fundamental observation that motivates many of the chapters here is that the fossil record demonstrates what has been called stasis. Niles Eldredge argues that the fossil record is typified by there being approximate morphological constancy with time with few signs of species-wide changes in form. Furthermore, the morphological changes that are seen are thought to be associated with speciation. The discrepancy between observed 'stasis' and neo-Darwinism is often overstated. No neo-Darwinian would be surprised that rates of morphological evolution are not clock-like, and it is sometimes hard to discern whether stasis implies any more than that there are variations in the rate of morphological evolution with time. However, the problem is that once stasis has been taken as the major evolutionary pattern to be explained, theoretical approaches to evolution end up being judged by their ability to predict this picture of a constant crop of morphologically invariant species, with new species occasionally being created by a quasi-sudden change in morphology.

The question therefore becomes whether there is anything in evolutionary process that would lead to these dynamics. In reality, as pointed out by Bergman and Feldman here, explaining stasis and sudden change in the fossil record is extraordinarily easy using neoDarwinian population genetics - all one needs is a mutation affecting morphology arising and spreading to fixation, either by selection or by drift. This is neoDarwinian gradual change in that the allele frequency changes gradually, but the total time taken for a substitution is instantaneous on the palaeontological level. However, many authors here seek more complex models that predict intermittent genetic change. The favourite is to suppose that there are epistatic relationships between genotype and fitness, as are seen in Sewall Wright's adaptive landscapes. In Wright's vision of such adaptive landscapes, populations can occupy different adaptive peaks, movements between which are difficult since a fitness valley must be crossed. Gavrilets here argues, however, that with a multidimensional genetic space, populations will still seek local optima, but, given the multidimensionality, and what he calls 'holey adaptive landscapes', there will be neutral paths through genotype space, which will be followed with low probability and which will give comparatively sudden changes in multilocus genotype. Clearly, the predicted outcome is inconsistency in evolutionary rates with time. Crutchfield's model of epochal evolution, while less formal genetically, shares the feature of neutral paths through a multidimensional space, until a population encounters a new attractor which will lead it rapidly through a process of hill climbing to a new improved optimum.

Thus, there appears to be an implicit synthesis between the empirical observations of sudden changes in fossil morphologies in morphological space, and theoretical sudden changes in genotypes in genotype space. The changes in fossil morphologies are inferred to correspond to speciations, as are rapid movements to new adaptive peaks in genotype space. However, I believe that this implicit synthesis is fundamentally wrong. In particular, it is wrong in that individual species are thought to represent local fitness optima in a mapping of fitness onto genotype space that is itself invariant. Much more probable is that the fitnesses of individual genotypes generally depend on the phenotypes of the organisms present in the environment. For example, there is no resolution, in a model in which fitness follows from genotype alone, of the issue how two species, with different phenotypes, are able to stably coexist in an ecological community. If these species occupy different peaks in a constant adaptive landscape, they will have different fitnesses, and one should replace the other. Clearly, such stability is impossible unless the 
absolute fitnesses of individuals depend on the other members of the biological community. It cannot realistically be assumed that there exists a multidimensional genotype space, with each point in the space having a constant fitness, independently of which individuals have which genotypes. There is a wholly different view of species based on ideas of ecological niches, which create independent density regulation of different species. In this view, changes in phenotype can create clear morphological breaks that constitute species, not because the epistatic interactions between genes result in peak shifts, but because the phenotypic changes reduce competition between the two groups such that they can stably coexist.

JFY Brookfield Institute of Genetics, University of Nottingham, Queens Medical Centre, Nottingham, NG7 2UH, UK E-mail: john.brookfield@nottingham.ac.uk 